\title{
Exclusion limits from data of directional Dark Matter detectors
}

\author{
J. Billard * F. Mayet, and D. Santos \\ Laboratoire de Physique Subatomique et de Cosmologie, \\ Université Joseph Fourier Grenoble 1, CNRS/IN2P3, \\ Institut Polytechnique de Grenoble, Grenoble, France
}

(Dated: October 22, 2018)

\begin{abstract}
Directional detection is a promising search strategy to discover galactic Dark Matter. Taking advantage on the rotation of the Solar system around the Galactic center through the Dark Matter halo, it allows to show a direction dependence of WIMP events. Even though the goal of directional search is to identify a WIMP positive detection, exclusion limits are still needed for very low exposure with a rather large background contamination, such as the one obtained with prototype experiments. Data of directional detectors are composed of energy and a 3D track for all recoiling nuclei. However, to set robust exclusion limits, we focus on the angular part of the event distribution, arguing that the energy part of the background distribution is unknown. As the angular distributions of both background and WIMP events are known, a Bayesian approach to set exclusion limits is possible. In this paper, a new statistical method based on an extended likelihood is proposed, compared to existing ones and is shown to be optimal. Eventually, a comprehensive study of the effect of detector configurations on exclusion limits is presented. It includes the effect of having or not sense recognition, a finite angular resolution, taking into account energy threshold as well as some astrophysical uncertainties.
\end{abstract}

PACS numbers: 95.35.+d, 14.80.-j

\section{INTRODUCTION}

In the context of direct detection of non-baryonic Dark Matter (WIMP), an alternative strategy to massive detectors [1 3], aiming at high background rejection and planning to scale up to ton-scale, is the development of detectors providing an unambiguous positive WIMP signal. This can be achieved by searching for a correlation of the WIMP signal with the solar motion around the galactic center, observed as a direction dependence of the WIMP flux [4], coming from $\left(\ell_{\odot}=90^{\circ}, b_{\odot}=0^{\circ}\right)$ in galactic coordinates, which happens to be roughly in the direction of the Cygnus constellation. This is generally referred to as directional detection of Dark Matter and several projects of detector are being developed for this goal [5-9].

The major advantage of directional detection is the fact that the angular distribution of WIMP events is pointing toward the Cygnus constellation while the background one is isotropic. This opens the possibility to really identify the WIMP signal as such. The Discovery parameter in this case is a signal pointing toward Cygnus as emphasized in [10, 11] since it cannot be mimicked by background. Recently, a statistical map-based analysis has been developed [10], showing the possibility to extract from data samples of forthcoming directional detectors, both the main direction of the incoming events, thus proving the galactic origin of the signal, and the number of WIMP events contained in the map thus constraining the WIMP-nucleon cross section.

*Electronic address: billard@lpsc.in2p3.fr
Even though the goal of directional experiments is to identify a WIMP positive detection, exclusion limit is still needed, for very low exposure with a rather large background contamination, such as the one obtained with prototype directional experiments.

The directional insensitive direct detection strategy is based on the measurement of the recoil energy $\left(d N / d E_{R}\right)$ and a dedicated statistical method has been developed 12] to optimize the exclusion limit obtained from a given set of data. In addition to the recoil energy, directional detectors also provide, for each track, the scattering angle $(\Omega)$. Recently a $2 \mathrm{D}$ extension of the previous method has been proposed 13, 14]. It is based on the doubledifferential spectrum $\left(d^{2} N / d E_{R} d \Omega\right)$ and allows to account for all information given by a directional detector. As a matter of fact, the energy spectrum of the background is unknown 15, 16], while its angular spectrum is expected to be isotropic in the galactic rest frame. We take advantage on this point by proposing a new method, taking into account only the angular part of the spectrum $(d N / d \Omega)$ in a given recoil energy range. As no assumption on the background energy dependence is needed, robust and conservative exclusion limits may be provided. We also argue that upcoming directional data may potentially be contaminated with a rather large amount of background events and this method is intended to cope with low signal to noise ratio, when identification is not possible [10]. This method is based on a likelihood analysis of events that are supposed to be composed of both background and WIMP events.

The paper is organized as follows. In section III we will first introduce the directional detection framework. In section [II] we will introduce the different statistical methods used to set WIMP-nucleon cross section limits : the existing one (Poisson, Maximum Gap [12]) and a 
new one, referred to as the Directional Likelihood method. They will be compared in section [IV for various detector configurations such as ideal detector, with or without sense recognition, finite angular resolution, and threshold effect. It allows to evaluate the impact of experimental uncertainties on each method. The effect of some astrophysical uncertainties like the WIMP local velocity dispersion and the local dark matter density $\rho_{0}$ will also be discussed.

\section{DIRECTIONAL DETECTION: EXPERIMENTAL AND THEORETICAL FRAMEWORK}

Several directional detectors are being developed and/or operated: MIMAC [5], DRIFT [6], DM-TPC [7] and NEWAGE [8]. A detailed overview of the status of experimental efforts devoted to directional Dark Matter detection is presented in [9]. Directional detection of Dark Matter requires track reconstruction of recoiling nuclei down to a few $\mathrm{keV}$. This can be achieved with low pressure gaseous detectors [17] and several gases have been suggested: $\mathrm{CF}_{4},{ }^{3} \mathrm{He}+\mathrm{C}_{4} \mathrm{H}_{10}$ or $\mathrm{CS}_{2}$. Both the energy and the track of the recoiling nucleus need to be precisely measured. Ideally, recoiling tracks should be $3 \mathrm{D}$ reconstructed as the required exposure to reject the background hypothesis is decreased by an order of magnitude between the 2D readout and 3D readout [18]. Sense recognition of the recoil track (head-tail) is also a key issue for directional detection [19 21] and its impact on exclusion limits calculation will be discussed here. The study is done for an ideal, but realistic, detector which could be within reach in a few years. We consider a 10 $\mathrm{kg} \mathrm{CF}_{4}$ detector, operated at 50 mbar and allowing 3D reconstruction of recoiling tracks. A recoil energy range $\left[E_{R_{1}}, E_{R_{2}}\right]$ is chosen between 5 and $50 \mathrm{keV}$. The lower bound of the energy range is due to the threshold ionization energy taking into account the quenching factor. As most of the WIMP events are concentrated at low recoil energy, an upper bound is chosen to limit the background contamination of the data. Indeed, for a WIMP mass of $100 \mathrm{GeV} \cdot \mathrm{c}^{-2}, 70 \%$ of the recoils are between $5 \mathrm{keV}$ and $50 \mathrm{keV}$ and only $10 \%$ are above $50 \mathrm{keV}$ in the case of an escape velocity taken as $v_{\text {esc }}=\infty$ and a form factor $F\left(E_{R}\right)$ approximated to one [45]. Thus, increasing the upper bound would lead to a potentially weaker signal to noise ratio. In section [V] we will study the effect of having or not sense recognition, degrading the angular resolution as well as varying the energy threshold.

Through this study, we focus on the simplest model for the Milky Way halo : the isotropic isothermal sphere in which the WIMP velocity follows a Maxwellian distribution defined in the laboratory rest frame as

$$
f(\vec{v})=\frac{1}{\left(2 \pi \sigma_{v}^{2}\right)^{3 / 2}} \exp \left(-\frac{\left(\vec{v}+\vec{v}_{\odot}\right)^{2}}{2 \sigma_{v}^{2}}\right)
$$

with a dispersion $\sigma_{v}=v_{0} / \sqrt{2}$ where $v_{0}=220 \mathrm{~km} . \mathrm{s}^{-1}$ is the circular speed at large radii. Effect of uncertainties on $v_{0}$, i.e on the local WIMP velocity dipersion, on exclusion limits will be discussed in sec. IVE We consider a detector velocity equal to the tangential component of the Sun's motion around the Galactic center $v_{\odot}=220 \pm 20$ $\mathrm{km} . \mathrm{s}^{-1}$, neglecting the Sun's peculiar velocity and the Earth's orbital velocity about the Sun. As a matter of fact, their contribution to the detector velocity is smaller than the uncertainty on $v_{\odot}$ [22]. Using the galactic coordinates $(\ell, b)$, the WIMP velocity is written in the galactic rest frame as :

$$
\vec{v}=v(\cos \ell \cos b \hat{x}+\sin \ell \cos b \hat{y}+\sin b \hat{z})
$$

where $\hat{x}$ points toward the galactic center, $\hat{y}$ in the direction of the solar motion and $\hat{z}$ toward the galactic north pole. The WIMP-induced recoil events are then computed by generating random incident WIMP velocities from $v f(\vec{v})$ and assuming an isotropic elastic scattering in the center of mass frame. Then, the recoil energy $\left(E_{R}\right)$ is given, in the laboratory frame, by :

$$
E_{R}=\frac{2 m_{\chi}^{2} m_{N} v^{2}}{\left(m_{\chi}+m_{N}\right)^{2}} \cos ^{2} \theta_{R}
$$

with $m_{\chi}$ the WIMP mass, $m_{N}$ the mass of the target and $\theta_{R}$ the recoil angle with respect to the WIMP direction. Thus, this Monte Carlo WIMP event generation can be used both for simulating experiments and for evaluating theoretical angular distributions. Then, in the case of the binned version of the Directional Likelihood method, described in sec. III 1 1 the theoretical event distributions of WIMP events $S$ and background events $B$ are estimated using $10^{8}$ Monte Carlo generated events. In the case of the background, the events are randomly generated according to an isotropic distribution. However, we have also developed an unbinned definition of our new $D i$ rectional Likelihood method (see sec. IIIC 1). In this case, the $S$ and $B$ distributions are analytically calculated. Indeed, as the spherical isothermal halo model leads to a WIMP-induced recoil distribution which presents a spatial distribution of events with an axial symmetry along the $\hat{y}$ axis, we will use the $\cos \gamma$ distribution of the events with $\cos \gamma=\hat{y} . \hat{r}, \hat{r}$ being the recoil direction in the galactic coordinates. In order to evaluate $S(\cos \gamma)$ (see sec. IIIC 1), we used the following theoretical distribution [4]:

$$
\frac{d R}{d \cos \gamma}=\kappa \int_{E_{R_{1}}}^{E_{R_{2}}} \exp \left[-\frac{\left(v_{\odot} \cos \gamma-v_{\min }\right)^{2}}{2 \sigma_{v}^{2}}\right] d E_{R}
$$

$\kappa$ is a normalization factor, $v_{\min }=\sqrt{m_{N} E_{R} / 2 m_{r}^{2}}$ corresponds to the minimal WIMP velocity required to produce a nuclear recoil of energy $E_{R}$ and $m_{r}$ is the WIMP-nucleus reduced mass. Moreover, it is worth noticing that due to this axial symmetry, there is no loss of information when looking at 1D angular distribution $(\cos \gamma)$ rather than 2D angular spectrum $(\ell, b)$. The theoretical background distribution $B(\cos \gamma)$ 
corresponds to a flat distribution as the background is taken to be isotropic.

The expected number of WIMP events $\mu$ is calculated by integrating the theoretical recoil energy distribution over the experimental energy range $E_{R_{1}}$ to $E_{R_{2}}$ :

$$
\mu(\sigma)=\frac{\sigma \rho_{0} \xi}{2 m_{r}^{2} m_{\chi}} \int_{E_{R_{1}}}^{E_{R_{2}}} F^{2}\left(E_{R}\right) \int_{v_{\min }}^{\infty} \frac{f(\vec{v})}{v} d^{3} v,
$$

with $\sigma$ the WIMP-nucleus elastic scattering cross section, $\rho_{0}$ the local dark matter density and $\xi$ the exposure. In the following we define $\sigma_{0}$ the WIMP-nucleon cross section directly related to $\sigma$ in the pure-proton approximation, see sec. V.

\section{WIMP-NUCLEON CROSS SECTION LIMITS CALCULATION METHODS}

In this section, we present the existing statistical methods, Poisson and Maximum Gap [12], and we propose a new one dedicated to directional detection, referred to as Directional Likelihood method.

\section{A. Poisson method}

The simplest way to derive a cross section upper limit from a given experimental dataset, is to use the Poisson method. This is the most conservative approach because it assumes no knowledge on neither the background nor the WIMP event distribution. Each recorded event is interpreted as a WIMP one. Hence, the aim of this method is to define the value of $\sigma_{0}$ corresponding to a number of signal events, $\mu_{\text {exc }}$, which is excluded at a given confidence level (CL) according to the Poisson distribution. The confidence level is then given by $1-\alpha(\mu)$, with

$$
\alpha(\mu)=e^{-\mu} \sum_{m=0}^{N} \frac{\mu^{m}}{m !}
$$

and $\mathrm{N}$ is the total number of events contained in a dataset. Then, the excluded value of $\sigma_{0}$ at $90 \% \mathrm{CL}$ is deduced from the value of $\mu_{\mathrm{exc}}$, which satisfies $1-\alpha\left(\mu_{\text {exc }}\right)=$ 0.9 , using equation (4).

As in the Poisson method all events are considered as a WIMP signal, any background observed events will lead to overly estimated upper limits. Even though it has been shown [12, 13] that, in the case of a pure WIMP signal, the Poisson method gives more restrictive upper limits than the Maximum Gap method, in this paper we are only interested in a dataset without WIMP events or with a large background contamination, which seems to be closer to real data from upcoming directional detectors [9]. We argue that in the case of a pure WIMP signal, directional detection should lead to an identification of a positive WIMP detection, using the methods presented in [10, 11].

\section{B. 1D Maximum gap method}

In order to deal with an unknown background contamination, which is the case with direction-insensitive direct detection (i.e. measuring only the energy of the recoil), two statistical methods have been proposed by S. Yellin [12] : "the maximum gap" and "the optimal interval". In this paper, we define a modified version of the maximum gap method applied to $1 \mathrm{D}$ directional data, i.e. the angular part of the spectrum $(d R / d \cos \gamma$ distribution, see sec. (II). Then, for a given value of $\sigma_{0}$ and a given dataset of $\mathrm{N}$ recorded events, we obtain a set of $(\mathrm{N}+1)$ gaps $x_{i}$ which are calculated as [12]:

$$
x_{i}=\int_{\cos \gamma_{i}}^{\cos \gamma_{i+1}} \frac{d R}{d \cos \gamma}\left(\sigma_{0}\right) d \cos \gamma
$$

with $\cos \gamma_{0}=-1$ and $\cos \gamma_{N+1}=1$ which are the lower and upper bounds of the $d N / d \cos \gamma$ distribution. The maximum gap $x$ is then defined as the largest $x_{i}$ obtained for a given experiment. The interest of the "maximum gap" method is the fact that the distribution of $x$ is independent of the theoretical distribution of the events. It depends only on $\mu$ and not on the shape of the event distribution. We refer the reader to [12] for a detailed discussion on setting limits using the Maximum Gap method.

We do not use full information from the datasets, i.e. $d^{2} R / d E_{R} d \cos \gamma$, as in [13], since the performance of the method strongly depends on the assumed background distributions. As the energy distribution of background events is unknown, we prefer to consider only the angular part of the distribution for which the background is known to be isotropically distributed, leaving aside the energy information of recorded events.

\section{Directional Likelihood methods}

As stated above, we restrict the information to the angular distribution of the events. In this case, the background is well understood and then, we have a theoretical distribution for both WIMP events and background ones thus allowing a Bayesian calculation of exclusion limits using a likelihood analysis.

\section{Definition}

The Directional likelihood method is based on a recent paper [10] which aims at distinguishing a genuine WIMP signal in a directional dataset. This likelihood definition allows to recover the main direction of the recoils and the number of WIMP events contained in the recoil map in order to identify a detection of particles from the galactic Dark Matter halo and to evaluate its significance. It has been shown that this analysis tool gives satisfactory results on a large range of exposure 
and background contamination levels. But, for very low number of WIMP events and for very large background fractions, when this method failed at recognizing a WIMP detection, obviously an exclusion limit should be derived. This will be the case for the very first results of directional detectors with low exposures.

The total number of recorded events $\mathrm{N}$ is considered as the sum of $n_{s}$ signal events and $n_{b}$ background events, i.e. $N=n_{s}+n_{b}$, where both $n_{s}$ and $n_{b}$ can be regarded as Poisson variables with means $\mu_{s}$ and $\mu_{b}$ respectively. The fact that both signal and background angular spectra are well known allows to derive upper limits using the Bayes' theorem. In the case of flat priors for both $\mu_{s}$ and $\mu_{b}$, and taking the evidence as a normalization factor, it is reduced to

$$
P\left(\mu_{s}, \mu_{b} \mid \vec{D}\right) \propto \mathscr{L}\left(\mu_{s}, \mu_{b}\right)
$$

where $\vec{D}$ refers to the characteristics of the data, as the total number of recorded events $N$, their direction and their energy.

However, in order to incorporate in the likelihood definition both the information contained in the measured angular spectrum and the fact that $N$ is a Poisson variable of mean $\mu_{N}=\mu_{s}+\mu_{b}$, an extended likelihood function is used:

$$
\mathscr{L}\left(\mu_{s}, \mu_{b}\right)=\frac{\left(\mu_{s}+\mu_{b}\right)^{N}}{N !} e^{-\left(\mu_{s}+\mu_{b}\right)} \times L\left(\mu_{s}, \mu_{b}\right)
$$

In the following, two different definitions of $L\left(\mu_{s}, \mu_{b}\right)$ are proposed, in the case of binned or unbinned data.

When using binned data, the following definition of $L\left(\mu_{s}, \mu_{b}\right)$ is used :

$$
L\left(\mu_{s}, \mu_{b}\right)=\prod_{i=1}^{N_{\text {pixels }}} P\left(\frac{\mu_{s}}{\mu_{s}+\mu_{b}} S_{i}+\frac{\mu_{b}}{\mu_{s}+\mu_{b}} B_{i} \mid M_{i}\right)
$$

where $M$ corresponds to the observed recoil map, $S$ and $B$ refer to the signal (WIMP) and background theoretical distributions (see sec. III). The probability $P$ is then evaluated at each bin with a poissonian distribution. Here the bins are the pixels of the observed recoil map represented in galactic coordinates. An angular resolution of $15^{\circ}$ (FWHM) is chosen [23], corresponding to a number of equal area pixels of $N_{\text {pixels }}=768$.

Unbinned data are often preferred and we propose a unbinned version of the definition of $L\left(\mu_{s}, \mu_{b}\right)$, defined as :

$$
L\left(\mu_{s}, \mu_{b}\right)=\prod_{i=1}^{N}\left(\frac{\mu_{s}}{\mu_{s}+\mu_{b}} S\left(\cos \gamma_{i}\right)+\frac{\mu_{b}}{\mu_{s}+\mu_{b}} B\left(\cos \gamma_{i}\right)\right)
$$

with $S$ and $B$ the theoretical distributions, $d R / d \cos \gamma$ (see sec. II), of WIMP and Background events respectively.
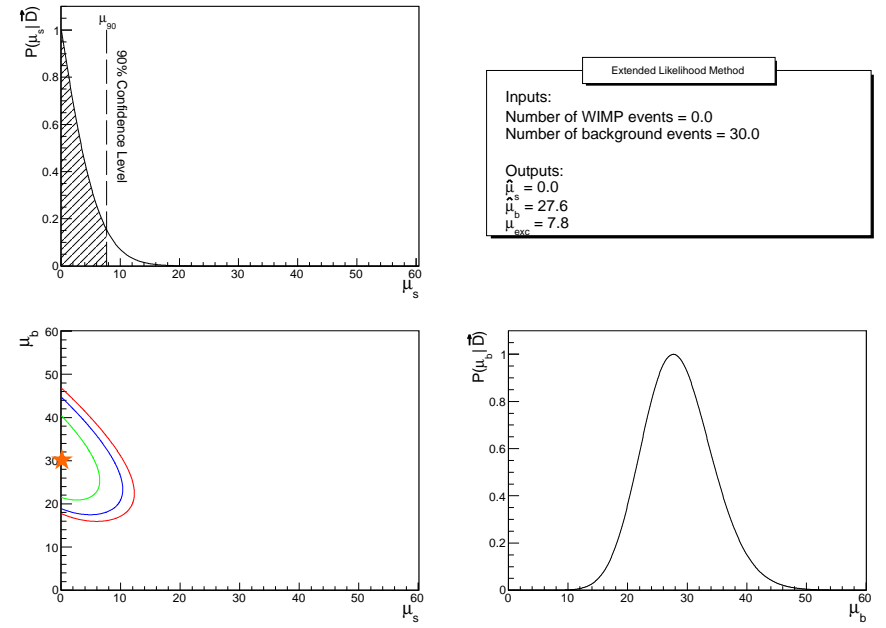

FIG. 1: Representation of $\mathscr{L}\left(\mu_{s}, \mu_{b}\right)$ in the case of 0 expected WIMP events and 30 expected background events. The lower left panel presents the 2 dimensional likelihood distribution with the $68 \%, 90 \%$ and $95 \%$ CL contours. The orange star represents the input values of $\mu_{s}$ and $\mu_{b}$. The left upper panel and the right lower panel represents the marginalized distributions $P\left(\mu_{s} \mid \vec{D}\right)$ and $P\left(\mu_{b} \mid \vec{D}\right)$, respectively.

\section{Setting exclusion limits using $P\left(\mu_{s}, \mu_{b} \mid \vec{D}\right)$}

Using a Bayesian approach, the probability density function of the parameter of interest $\mu_{s}$ can be derived by marginalizing $P\left(\mu_{s}, \mu_{b} \mid \vec{D}\right)$ over the parameter $\mu_{b}$. Then, the value of $\mu_{\text {exc }}$ is obtained by solving:

$$
\int_{0}^{\mu_{\mathrm{exc}}} P\left(\mu_{s} \mid \vec{D}\right) d \mu_{s}=0.9
$$

Thus, the value of the excluded cross section at $90 \% \mathrm{CL}$ is deduced from $\mu_{\text {exc }}$ using equation (4). As an illustration of the method, figure 1 presents the results from the calculation of $\mathscr{L}\left(\mu_{s}, \mu_{b}\right)$ in a working example with 0 expected WIMP event and 30 expected background events. The probability density functions of $\mu_{s}$ and $\mu_{b}$ are derived from the marginalization of the 2 dimensional likelihood over the $\mu_{b}$ and $\mu_{s}$ parameters respectively. The calculation of $\mu_{\text {exc }}$ is also illustrated and represented on the left upper panel of figure 1. Hence, on this example of 30 simulated events, the method allows to exclude at $90 \%$ CL a number of WIMPs greater than 7.8. At last, a strong correlation $(\rho \sim-1)$ between the parameters $\mu_{s}$ and $\mu_{b}$ is observed on the 2 dimensional representation of $\mathscr{L}\left(\mu_{s}, \mu_{b}\right)$ and is explained by the fact that $n_{s}=N-n_{b}$.

\section{Comparison between binned and unbinned likelihood definitions}

When using binned data, bias can be introduced in the analysis and thus, whenever possible, unbinned data 
analysis are preferred. For each configuration and each result presented in this paper, both binned and the unbinned analyses have been done, using the proper likelihood definition. No difference between them has been seen, due to the fact that, in our case, the binning (pixels area) is thin enough and the theoretical distributions are smooth and much wider than the pixels. As the effect of bining is null, using binned data do not introduce any bias in the calculation of $P\left(\mu_{s}, \mu_{b} \mid \vec{D}\right)$ and then in the estimation of the excluded cross section. Hence, only the results obtained with the binned likelihood definition, referred to as the Directional Likelihood method, is presented hereafter.

\section{Discussion on limit setting methods}

In order to evaluate the frequency distributions of the excluded cross section at 90\% CL, see figure 2, we used 10,000 toy Monte Carlo experiments for each case and for the three statistical methods.

From each distribution, we can derive the median value of the excluded WIMP-nucleon cross section $\sigma_{m e d}$ and the RMS given by the Poisson, Maximum Gap and the Likelihood methods. As an example, on figure 2 we consider a case with 10 expected WIMP events and 10 expected background events. The coverage is defined as the fraction of experiments leading to an excluded cross section above the input value. The values deduced from the study are : $(99.99 \pm 1) \%,(98.76 \pm 1) \%$ and $(93.39 \pm 1) \%$ for the Poisson, Maximum Gap and likelihood methods respectively. The fact that the coverage is greater than $90 \%$ for the three different methods means that in the case of a background contamination, the excluded cross section is overly estimated. In the case of the Poisson method, this over estimation comes from the fact that each recorded event is considered as a WIMP event. Contrary to the Poisson method, the Likelihood and the Maximum Gap methods evaluates the agreement between the distribution of the observed events with the theoretical WIMP event distribution. The fact that the coverage of the Likelihood method is closer to $90 \%$ CL than the other one is explained by the incorporation of the $L\left(\mu_{s}, \mu_{b}\right)$ term in the extended likelihood function which helps at discriminating WIMP events from the background ones contained in a given dataset. Thus, the Likelihood method is the most robust method in the case of a sizeable background contamination. Note that if the expected number of background events could be accurately estimated even with some uncertainties, it could be possible to consider a non constant prior $P\left(\mu_{b}\right)$ in order to improve the efficiency of the Likelihood method. However, as discussed in [12], as the number of expected background $\mu_{b}$ cannot be known without important uncertainties, it is not possible to use the Feldman-Cousins technique [24] and the ones presented in 25].

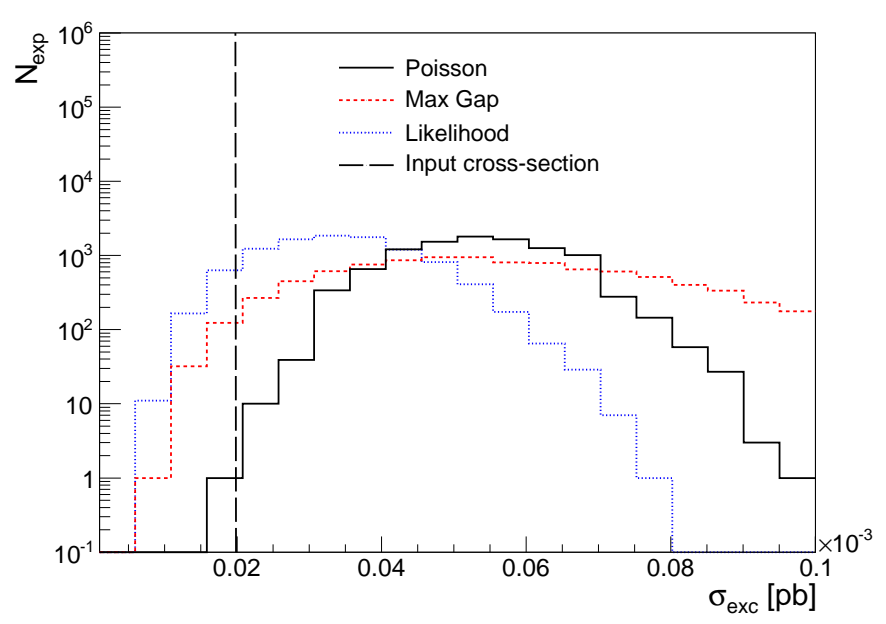

FIG. 2: Frequency distributions of the excluded cross section $\sigma_{\text {exc }}$ at $90 \%$ CL for each method with 10 expected WIMP events and 10 expected background events. The Poisson, Maximum Gap and Likelihood methods are represented by the black solid line, the red dashed line and the blue dotted line respectively. The coverage for each methods are (99.99 \pm $1) \%,(98.76 \pm 1) \%$ and $(93.39 \pm 1) \%$ respectively.

\section{COMPARISON OF THE EXCLUSION LIMIT METHODS}

As the statistical framework is being defined, we can compare the efficiency of the three methods. We focus on pure background or highly background-contaminated data, as it is the most realistic case for upcoming directional detectors, especially prototype experiments with low exposures. In the following, we evaluate with the three statistical methods, the effect of detector configurations on the exclusion limits, such as having or not sense recognition capability, having a low angular resolution and varying the energy threshold. The effects of astrophysical uncertainties on the calculation of cross section limits are eventually evaluated.

Unless otherwise stated, we consider a $\mathrm{CF}_{4}$ directional detector and a halo model as presented in section $\amalg$ with a total time exposure of 3 years and a WIMP mass taken to be $100 \mathrm{GeV} \cdot \mathrm{c}^{-2}$.

\section{A. Ideal detector}

First, an "ideal detector" is considered, meaning a detector allowing a 3D reconstruction of recoiling events with sense recognition and with perfect angular definition. The three methods are compared on two simulated datasets : pure background (fig. 31) and background events with 5 expected WIMP events (fig. (4). The first case reflects a scenario in which the exposure is not sufficient to access WIMP events due to a very low WIMP-nucleon cross section while in 
the second case a few WIMP events are obtained as the considered cross section was increased. Further an increase of the number of WIMP events is meaningless in the context of directional detection, as in such case a discovery could be done rather than an exclusion, as shown in [10]. Figure 3 and 4 present the excluded cross section $\left(\sigma_{m e d}\right.$ as defined in sec. IIID) obtained with the three different methods as a function of the number of expected background events.

In the pure background case (fig. 3), the three methods start with a common value at null event. Indeed, in such a case, the Maximum Gap and the Likelihood methods are identical to the Poisson one. Then, when no event is observed, an excluded value of $\mu_{\text {exc }} \simeq 2.3$ WIMP events at 90\% CL is deduced from the three methods. The excluded cross section obtained with the three methods start being different as the number of background events increases. The Likelihood method allows to obtain a much more restrictive exclusion limit than the two others, by a factor $\sim 4$ at high background contamination (30 events). As expected, increasing the number of background events degrades the upper cross section limit (i.e. increases the value of $\sigma_{m e d}$ ). This holds for the three methods, noticing that the Likelihood method is less sensitive to background contamination, as it takes full advantage on the knowledge of the expected WIMP and background angular distributions.

In the case of data populated with a few WIMP events, the excluded cross section evaluated with the Likelihood method is only slightly less restrictive than in the former case. The two Poisson curves (black solid lines) are exactly the same on figures 3 and 4 . Indeed, as the Poisson method is only dealing with the total number of events, the presence of WIMP events does not modify the exclusion limit. It can be noticed that below $\sim 10$

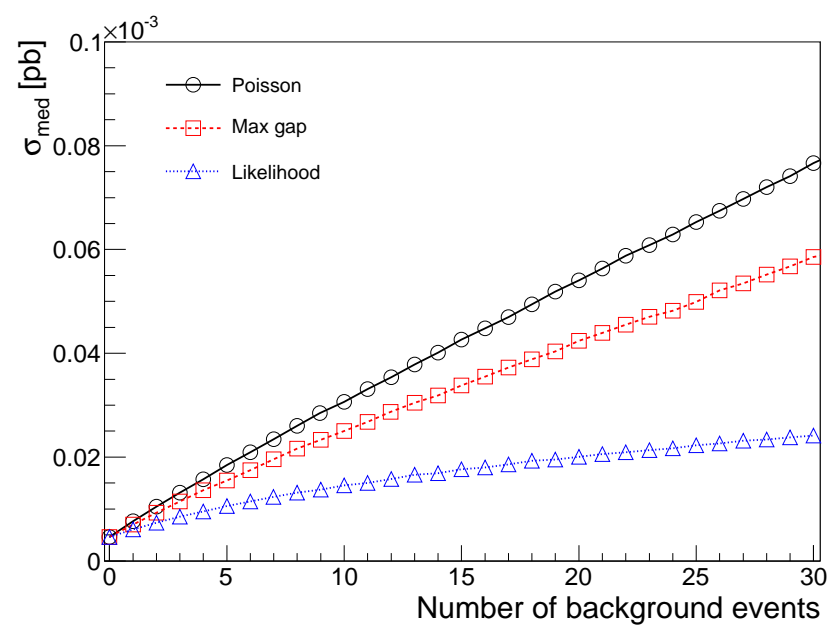

FIG. 3: The excluded cross section $\left(\sigma_{\text {med }}\right)$ as a function of the number of background events, in the case of a pure background, obtained by the three different statistical methods: Poisson (black solid line), Maximum Gap (red dashed line) and Likelihood (blue dotted line).

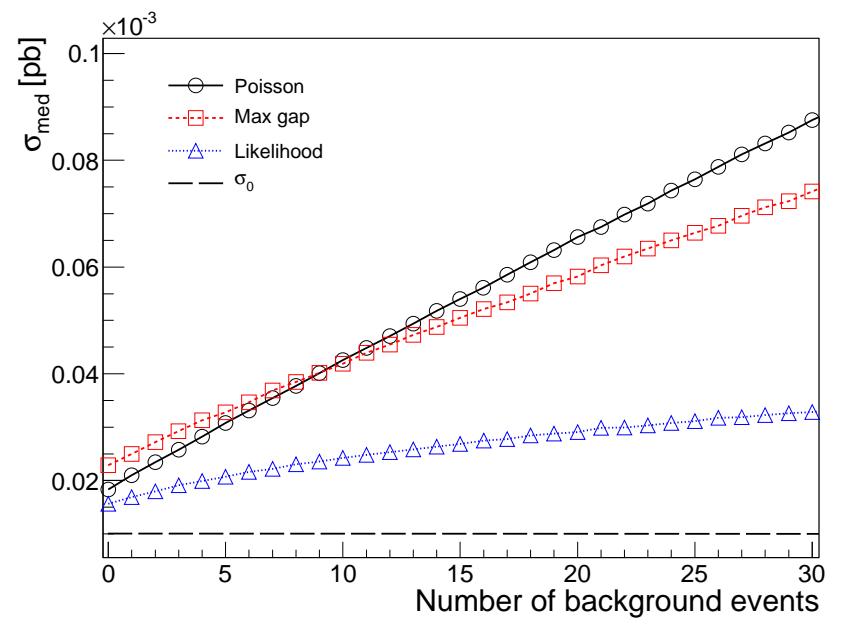

FIG. 4: The excluded cross section $\left(\sigma_{m e d}\right)$ as a function of the number of background events, in the case of 5 expected WIMP events, obtained by the three different statistical methods: Poisson (black solid line), Maximum Gap (red dashed line) and Likelihood (blue dotted line).

events, the Maximum Gap method gives worse results than the Poisson one. As a matter of fact, in the case of pure WIMP signal the Poisson method overcomes the Maximum Gap one, see sec. IIIA

As shown in [10], the directionality of the WIMP signal depends on the WIMP mass. Lighter is the WIMP, stronger is the angular anisotropy. Indeed, due to the finite energy range and the fact that low WIMP mass induces an energy distribution shifted to low energy, events above threshold $\left(E_{R_{1}}\right)$ are the one with the most directional feature (eq. (2)). The directionality evolves quickly at low masses and very slowly for masses heavier than a hundreds of GeV.c ${ }^{-2}$. A study of the effect of the WIMP mass on the cross section limit has been done. Figure 5 presents on the upper panel the cross section limit $\left(\sigma_{m e d}\right)$ as a function of the WIMP mass for the three methods considering 10 expected background events and one expected WIMP event. This highlights the fact that the Likelihood method gives the best result on the whole WIMP mass range. Moreover, we can notice that the cross section limit is more restrictive for masses lower than $\sim 30 \mathrm{GeV} . \mathrm{c}^{-2}$ due to the threshold of the detector. Indeed, at low masses, the energy distribution is more peaked at low energy leading to a loss of expected events and then to an increasing cross section limit. The lower panel of figure 5 presents the evolution of $\sigma_{m e d}$ divided by $\sigma_{0}$ (the input value to get 1 WIMP event). It allows to show that the cross section limit is enhanced by the directionality of the signal, at low WIMP masses $\left(\leq 100 \mathrm{GeV} \cdot \mathrm{c}^{-2}\right)$, for both the Maximum Gap and the Likelihood method. Increasing the WIMP mass does not affect the result as there are little differences in angular distributions for heavy WIMPs, as stated 

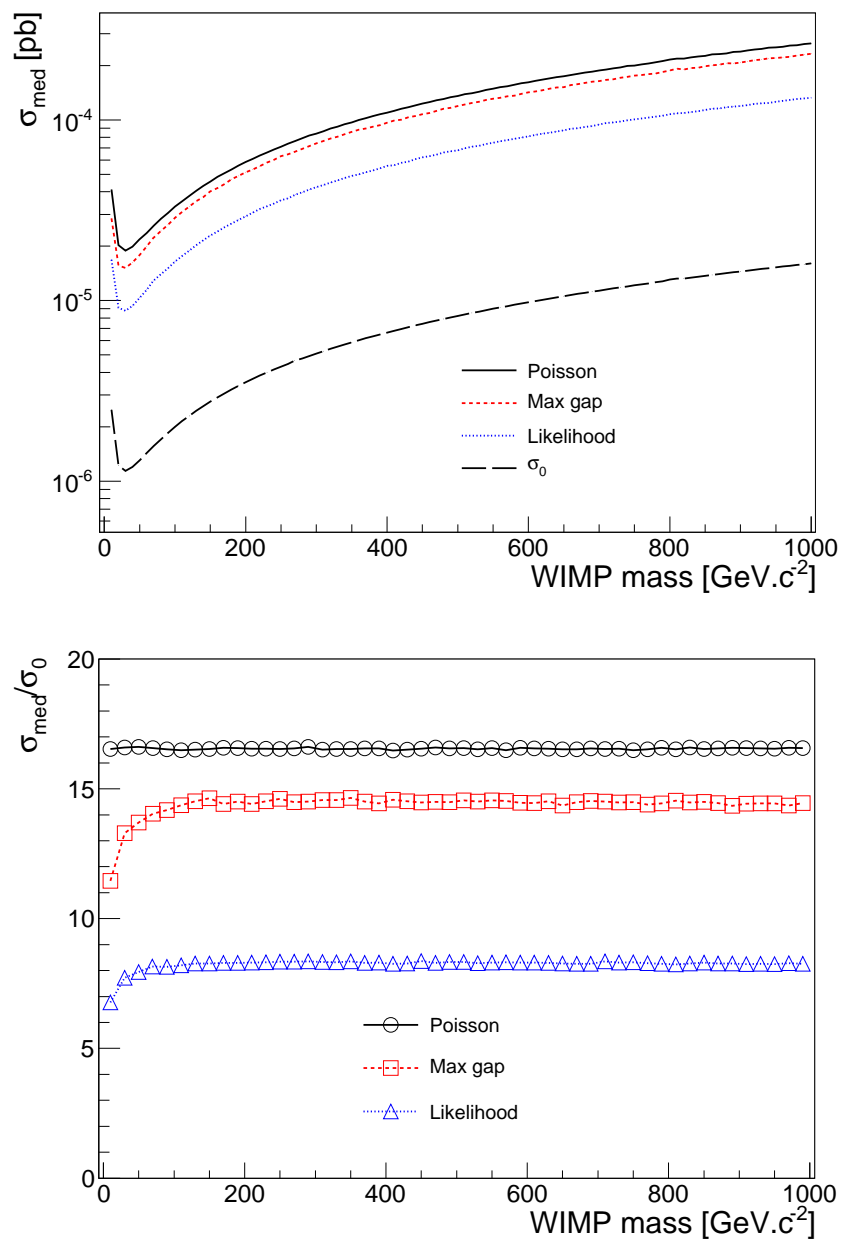

FIG. 5: Upper panel : cross section limit $\left(\sigma_{m e d}\right)$ as a function of the WIMP mass for the three methods considering 10 expected background events and one WIMP event. Lower panel : cross section ratio $\left(\sigma_{\text {med }} / \sigma_{0}\right)$ as a function of the WIMP mass. This highlights the fact that the cross section limit is enhanced by the directionality of the signal, at light WIMP masses $\left(\leq 100 \mathrm{GeV} \cdot \mathrm{c}^{-2}\right)$, for both the Maximum Gap and the Likelihood method.

above. As expected the Poisson method is not sensitive to the WIMP mass, as it handles only the total number of events recorded and not their angular distribution.

A key feature of the proposed Likelihood method is the fact that, contrary to the Maximum Gap method [12], it can deal with very large number of events. Hence, we study hereafter the evolution of $\sigma_{m e d}$ with the exposure, for a fixed WIMP mass $m_{\chi}=100 \mathrm{GeV} \cdot \mathrm{c}^{-2}$. Figure 6 presents the excluded cross section obtained with the Poisson and Likelihood methods as a function of the exposure. Curves are labeled according to the expected value of $\lambda$, defined as the WIMP event fraction in the dataset $\left(\lambda=\mu_{s} /\left(\mu_{s}+\mu_{b}\right)\right)$. To illustrate, we have chosen two different background rates $(2,20)$ evt $/ \mathrm{kg} /$ year and an input cross section $\sigma_{0}=10^{-5} \mathrm{pb}$ giving an expected rate of WIMP events equals to 0.2 evts $/ \mathrm{kg} /$ year. The Poisson

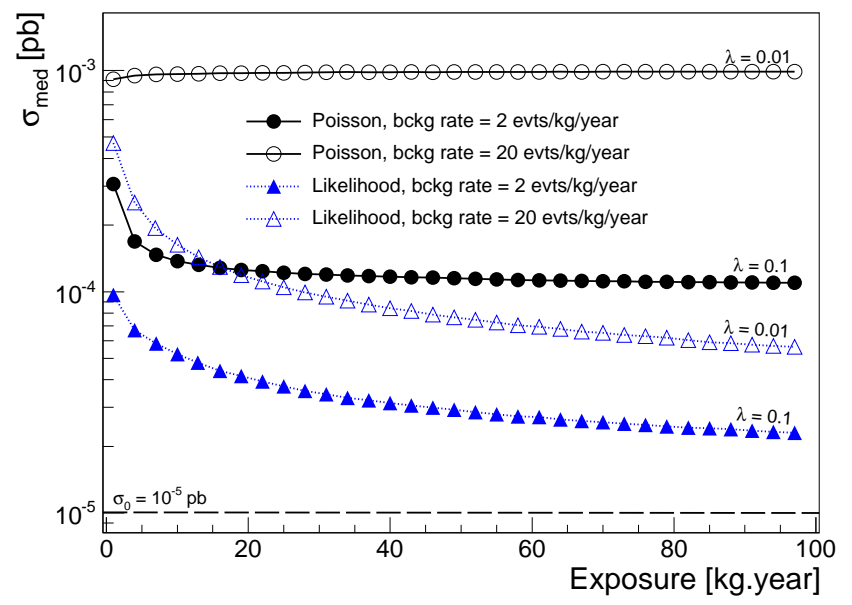

FIG. 6: The excluded cross section obtained with the Poisson and Likelihood methods as a function of the exposure. $\lambda$ is defined as the WIMP fraction in the events : $\lambda=\mu_{s} /\left(\mu_{s}+\mu_{b}\right)$. For this study the WIMP mass is fixed $m_{\chi}=100 \mathrm{GeV} . \mathrm{c}^{-2}$ and two different background rates are considered : $(2,20)$ evt $/ \mathrm{kg} /$ year. The input cross section is $\sigma_{0}=10^{-5} \mathrm{pb}$ giving an expected rate of WIMP events equal to 0.2 evts $/ \mathrm{kg} /$ year.

results (in black circles) are also represented in order to appreciate the efficiency of the Likelihood method (blue triangles).

With the Likelihood method, the cross section upper limit is still getting more restrictive, when increasing the exposure, especially in the case of highly backgroundcontaminated data. On the contrary, if the background fraction is too high $(\lambda \leq 0.01)$, the cross section limit given by the Poisson method remains flat as a function of the exposure. This is the proof that the Poisson method will not be able to deal with data contaminated by a large number of background events, as expected from upcoming directional prototype detectors. Then, the difference between the Poisson and the Likelihood method is about an order of magnitude on this range of exposure. But the most interesting point is the fact that only the Likelihood method will allow to set better exclusion limits with increasing exposure, even with critically low value of $\lambda$. As expected, when increasing the rejection power of the detector, i.e. increasing the expected value of $\lambda$, the two statistical methods (filled triangles and filled circles) are getting more efficient.

\section{B. Detector without sense recognition}

Even though several progresses have been done 19 21], sense recognition (so-called "Head-Tail" (HT) effect) remains a key and challenging experimental issue for directional detection of Dark Matter. In particular, it should still be demonstrated that sense recognition may be achieved at low recoil energy where most WIMP events reside. Hence, in the following we inves- 


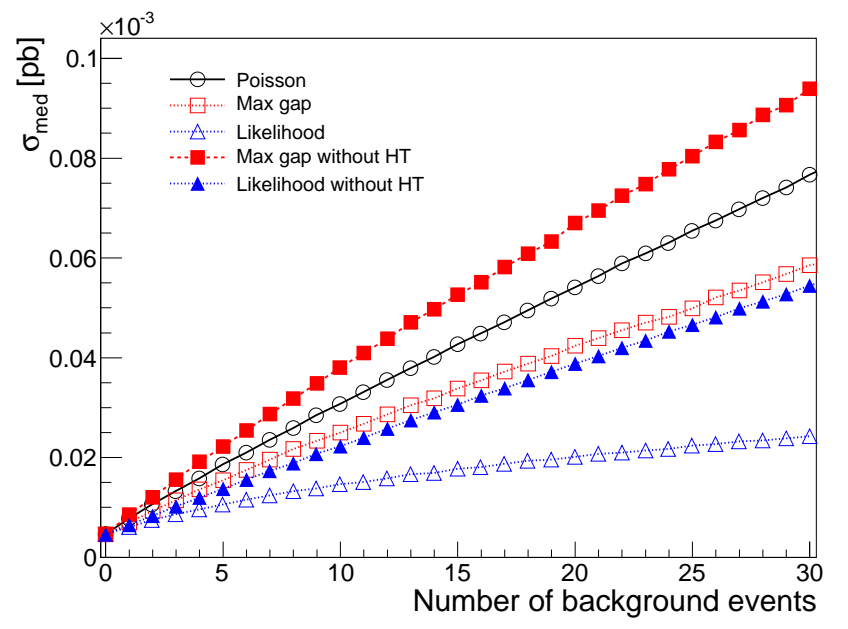

FIG. 7: The median upper limit cross section obtained by the three different statistical methods as a function of the number of background events in the case of pure background. The results without sense recognition are presented with filled markers while empty markers refer to a detector with sense recognition.

tigate the effect on exclusion limits of using a detector with no sense recognition. In such case, a recoil coming from $(\cos \gamma, \phi)$ cannot be distinguished from a recoil coming from $(-\cos \gamma, \phi+\pi)$. Then, we need to use axial data characterized by a direction $(|\cos \gamma|)$ and the directional event distribution becomes:

$$
\frac{d N}{d|\cos \gamma|}=\frac{d N(\cos \gamma)}{d \cos \gamma}+\frac{d N(-\cos \gamma)}{d \cos \gamma}
$$

We study the effect of no sense recognition in the case of pure background data and the result is presented on figure 7 To ease comparison, results with sense recognition are recalled (from figure 3). Still, the Likelihood method overcomes the two others and it appears that the absence of sense recognition only mildly alter the result (a factor of three at high background contamination). As expected, the Poisson method is insensitive to the absence/presence of sense recognition. Surprisingly, the Maximum Gap method becomes less competitive than the Poisson method in the case of no sense recognition. This is due to the fact that in this case, the expected WIMP distribution becomes less anisotropic and then it is getting closer to the expected background event distribution (isotropic). Indeed, as it has been shown in [12, 13], in the case where the difference between the background and the WIMP event distribution is very small, the Poisson method sets more competitive limits than the Maximum Gap one.

Taken at face value, this result suggests that sense recognition may not be so important for directional detection when trying to set exclusion limits. The difference between $100 \%$ sense recognition on the whole recoil energy range, which is obviously unrealistic, and no sense recognition is only minor (less than a factor of three at high background contamination). The worst case is indeed a partial sense recognition strongly depending on the recoil energy. In this case, we suggest not to consider this information to set exclusion limits. However, sense recognition remains a key issue worth investigating, for WIMP discovery which is the ultimate goal of directional detection.

\section{Detector with finite angular resolution}

Previous studies have been done in the case of a detector with a perfect angular resolution. However, realistic data of upcoming directional detectors should suffer from finite angular resolution. This is an intrinsic limitation of this detection strategy. Even if simulations show that straight line tracks may be 3D reconstructed with a rather small angular dispersion [26], realistic tracks in low pressure gaseous detectors would encounter a rather large "straggling" effect (angular dispersion). The lower is the recoil energy, the larger is the angular straggling. Hence, in the following we investigate the effect on exclusion limits of using a detector with a finite (realistic) angular resolution. Having a finite angular resolution means that a recoil initially coming from the direction $\hat{r}(\theta, \phi)$ is reconstructed as a recoil $\hat{r}^{\prime}\left(\theta^{\prime}, \phi^{\prime}\right)$. Then, the angular deviation between the initial recoil and the reconstructed one is given by:

$$
\Theta=\cos ^{-1}\left(\hat{r} \cdot \hat{r}^{\prime}\right)
$$

The $\hat{r}^{\prime}$ direction is characterized in the $\hat{r}$ frame by the angles $\Theta$ and $\Phi$ which are, for each event, randomly generated using the following probability density function $f(\Theta, \Phi)$ :

$$
f(\Theta, \Phi) \propto \sin \Theta \exp \left(-\frac{\Theta^{2}}{2 \sigma_{\Theta}^{2}}\right) \quad \begin{aligned}
& 0 \leq \Theta \leq \pi \\
& 0 \leq \Phi \leq 2 \pi
\end{aligned}
$$

In order to compute the theoretical angular distribution considering this finite angular resolution, we have done the convolution product of the initial angular spectra $d N / d \Omega$ with a smoothing gaussian function of width $\sigma_{\Theta}$ 27].

Figure 8 presents the cross section limit as a function of the angular resolution $\sigma_{\Theta}$ in the case of 10 expected background events and one expected WIMP event. It can be noticed that the Maximum Gap and the likelihood methods are only slightly dependent on the angular resolution of the detector. The deviation for the two methods is of the order of $30 \%$ from $\sigma_{\Theta}=0^{\circ}$ to $\sigma_{\Theta}=45^{\circ}$. Hence, as far as exclusion limits are concerned, the effect of angular resolution is relatively small but has to be correctly evaluated to set coherent upper limits. This outlines the need for detector commissioning, e.g. by using a neutron field [28].

As for the sense recognition study (sec. IVB) and for the same reasons, the Maximum Gap method is getting less 


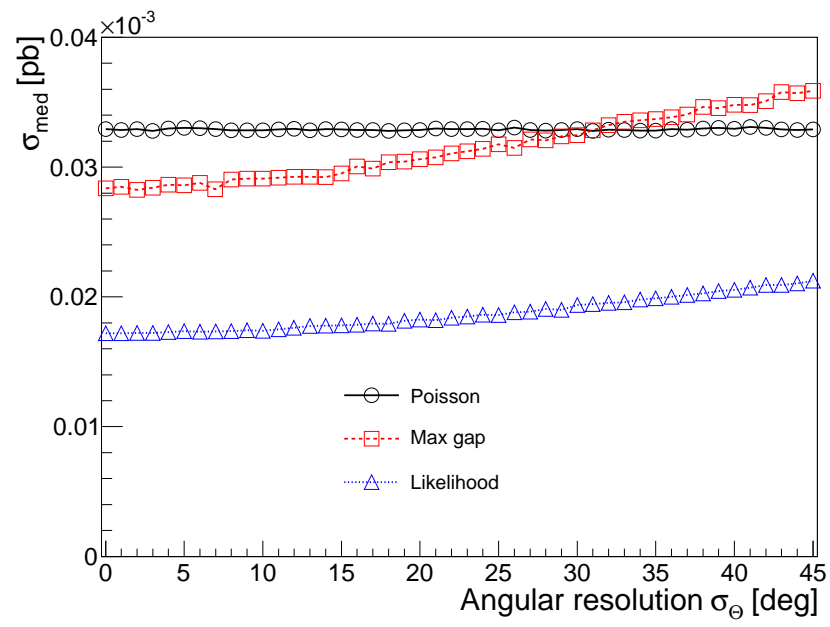

FIG. 8: The median upper limit cross section $\sigma_{\text {med }}$ obtained with the three methods as a function of the angular resolution. The study is done in the case of 10 expected background events and one expected WIMP event. The detector configuration is the following : sense recognition and energy threshold of $5 \mathrm{keV}$.

competitive than the Poisson method beyond $\sigma_{\Theta} \sim 30^{\circ}$. Indeed, the expected WIMP distribution and background are getting closer and then, as previously explained, the Poisson method is more competitive than the Maximum Gap one.

\section{Effect of energy threshold}

As for direction-insensitive direct detection, the energy threshold plays a key role for directional detection. It depends on the target and the quenching factor. It is defined as the minimal recoil energy for which both the energy and the 3D track are well reconstructed. Reducing the energy threshold leads to a higher expected number of WIMP events and hence a more restrictive exclusion limit. Figure 9 presents the exclusion limits obtained, evaluated by the three statistical methods and for three different values of the energy threshold $\mathrm{E}_{\mathrm{R}_{1}}$, in the case of 10 expected background events and one expected WIMP event. As the energy threshold $E_{R_{1}}$ is taken between 5 and $50 \mathrm{keV}$, the upper bound of the energy range $\mathrm{E}_{\mathrm{R}_{2}}$ is chosen as $200 \mathrm{keV}$ for this study. It can be noticed that the Likelihood method gives the best limit on the whole mass range and for the three threshold values. It is worth emphasizing that the effect of the energy threshold is very important even in the case of directional detection. Going from a $5 \mathrm{keV}$ to a $50 \mathrm{keV}$ energy threshold leads to a loss of about one order of magnitude in exclusion limits. The situation is even worse at low WIMP masses. This outlines the fact that, as far as exclusion limits are concerned, lowering the energy threshold remains the major experimental issue for upcoming directional detector projects.

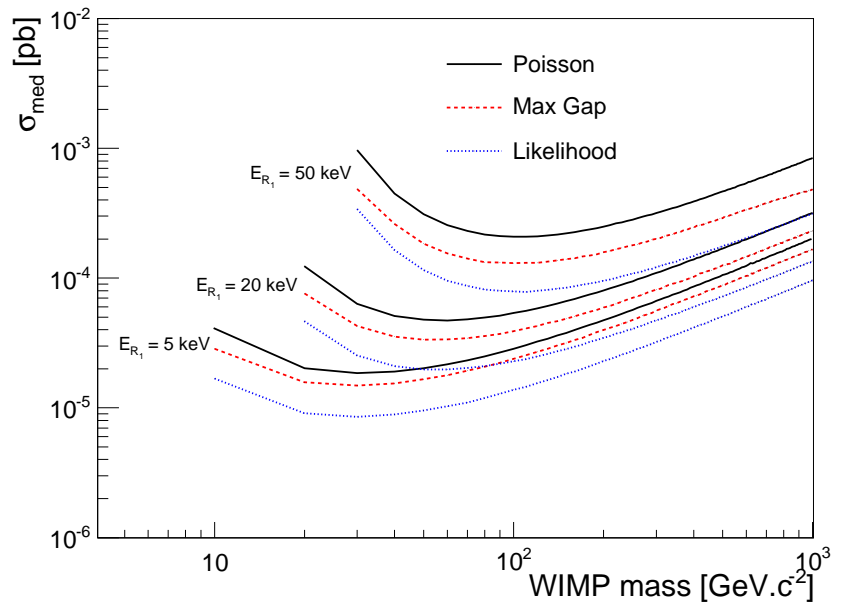

FIG. 9: Exclusion limits, WIMP-nucleon cross section as a function of the WIMP mass with the three methods and for three values of the energy threshold. The study is done in the case of 10 expected background events and one expected WIMP event. The upper bound of the energy range $E_{R_{2}}$ is chosen as $200 \mathrm{keV}$. The detector configuration is the following : perfect angular resolution and sense recognition.

\section{E. Impact on limits from astrophysical uncertainties}

The effect of astrophysical uncertainties on exclusion limits obtained with direction-insensitive direct detection has been studied in details in 29, 30]. In the following, we investigate the effect on directional detection.

Within the framework of a spherical isothermal halo model, two astrophysical parameters play a key role : the local dark matter density $\rho_{0}$ and the local WIMP velocity dispersion $v_{0}$.

The first one is usually quoted within the range $\rho_{0} \sim 0.2-0.8 \mathrm{GeV} . \mathrm{c}^{-2} . \mathrm{cm}^{-3}$ and the value $0.3 \mathrm{GeV} . \mathrm{c}^{-2}$ is used as a "standard" value for the sake of comparison of various direct detector results [31]. Recently, a value of the local Dark Matter density has been derived within the framework of a galaxy-model independent method [32]. The resulting local Dark Matter is $\rho_{0}=0.43 \pm 0.11 \pm 0.10 \mathrm{GeV} / \mathrm{c}^{2} / \mathrm{cm}^{3}$. As shown in [10], directional detection offers the possibility to constrain the WIMP-nucleon cross section, which can be relaxed into a constraint on $\rho_{0} \times \sigma$ as a function of the WIMP mass. Then, the measured value of $\rho_{0}$ [32], together with its uncertainty, shall be accounted for when presenting discovery regions. However, when setting limits, the value of $\rho_{0}$ does not change the shape of the exclusion limit, only its magnitude and with a negligible effect, owing to the orders of magnitude involved. In the following the so-called standard value is used.

The second astrophysical parameter to be carefully handled is the WIMP velocity dispersion $\sigma_{v}$ related to the asymptotic circular velocity $v_{0}$, for which the 


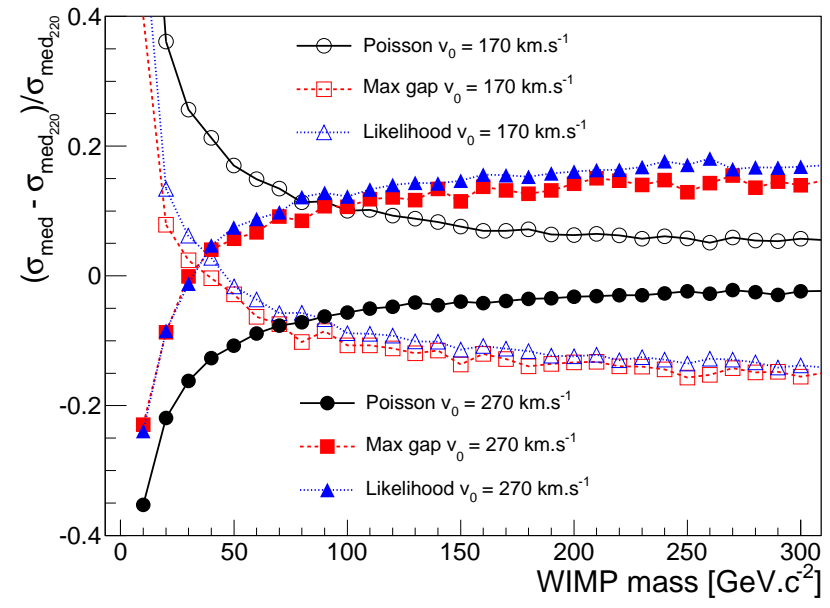

FIG. 10: Deviation of the median upper limit cross section $\left(\sigma_{m e d}\right)$ from the value $\sigma_{\text {med }_{220}}$ obtained with the standard value $v_{0}=220 \mathrm{~km} \cdot \mathrm{s}^{-1}$, as a function of the WIMP mass. The study is done, for the three methods, with two extreme cases : $170 \mathrm{~km} \cdot \mathrm{s}^{-1}$ and $270 \mathrm{~km} \cdot \mathrm{s}^{-1}$.

"standard" value is $220 \mathrm{~km} \cdot \mathrm{s}^{-1}$ [31]. As outlined in [29], recent determinations of its value span on a wide range and the impact for directionality has been studied, in the context of isotropy rejection [27]. In the following, we investigate the effect of its value on directional exclusion limits by studying two extreme cases (170 and $\left.270 \mathrm{~km} . \mathrm{s}^{-1}\right)$.

Figure 10 presents the deviation of the median upper limit cross section $\left(\sigma_{m e d}\right)$ from the value $\sigma_{\text {med }_{220}}$ obtained with the standard value $v_{0}=220 \mathrm{~km} \cdot \mathrm{s}^{-1}$ as a function of the WIMP mass. The study is done with a dataset of 10 background events and 1 WIMP event. For convenience, the Poisson case, represented by the black solid lines, is described first. As stated above, the Poisson exclusion limit is only sensitive to the total number of events and not to the directionality of the event angular distribution. When comparing low (170 km.s $\mathrm{s}^{-1}$, open circle) and high $\left(270 \mathrm{~km} . \mathrm{s}^{-1}\right.$, filled circle) values of $v_{0}$, three effects on the excluded cross section can be noticed. The first one is linked to the energy threshold. For a low value of $v_{0}$, the theoretical WIMP energy distribution is more peaked at low energy, leading to less events above the energy threshold. Hence, the cross section limit is higher in the case of $v_{0}=170 \mathrm{~km} \cdot \mathrm{s}^{-1}$ than for $v_{0}=270 \mathrm{~km} \cdot \mathrm{s}^{-1}$. A second effect is due to the WIMP mass. Indeed, the difference between low and high values of $v_{0}$ is increased for low WIMP masses. Again, this is due a shift of a fraction of WIMP events below the threshold at low WIMP masses.

When comparing with Maximum Gap and the likelihood methods, a third effect is introduced due to the directionality of the WIMP signal. The same general tendency is observed, but above a given WIMP mass $\left(\sim 40 \mathrm{GeV} / \mathrm{c}^{2}\right)$, the low $v_{0}$ exclusion limit becomes more restrictive. Indeed, a lower value of $v_{0}$ will induce a more directional WIMP event distribution as it is linked to the spread of the WIMP flux, see eq. 1. Then, the difference between the background and the WIMP signal is enhanced, leading to a better exclusion. To summarize, when the WIMP mass is sufficiently high, the effect of the directionality at low $v_{0}$ overcomes the induced loss of events previously described.

As a conclusion of this study, we can notice that the impact of uncertainties on the value of $v_{0}$ can lead to a deviation of the excluded cross section from $50 \%$ at low WIMP mass to $20 \%$ at large WIMP mass.

As stated above, for this first study of an optimal exclusion method for directional detection, the standard halo model (spherical isothermal) has been considered. This is indeed the reference model to compare Dark Matter experiments and associated exclusion methods. Anisotropy, triaxiality, and clumping in the WIMP velocity distribution would have an effect on directional detection signal. As far as directional exclusion limits are concerned, this will mildly affect the result presented in this paper. Indeed, only the theoretical WIMP distribution used for the directional likelihood method would be changed, though remaining directional (anisotropic) and then different from the background. However, the effect of non standard halo models (triaxial, with stochastic features or streams) will be addressed in a dedicated forthcoming paper [33].

\section{PROSPECTING EXCLUSION LIMITS WITH DIRECTIONAL DETECTION}

To end-up this study, we present projected exclusion limits for a forthcoming directional detector proposed by the MIMAC collaboration [5]. We consider a $10 \mathrm{~kg} \mathrm{CF}_{4}$ detector operated during $\sim 3$ years, allowing 3D track reconstruction, with a $10^{\circ}$ angular resolution and a recoil energy range $5-50 \mathrm{keV}$. In the following, a very low WIMP-nucleon cross section is considered, such as no WIMP event is expected during the acquisition time of the experiment. Note that a detector configuration has been chosen as an illustration, but the effect of the energy range, angular resolution has been shown previously.

Figure 11 presents the spin dependent cross section on proton $(\mathrm{pb})$ as a function of the WIMP mass $\left(\mathrm{GeV} / \mathrm{c}^{2}\right)$. Results are presented in the case of pure-proton approximation [34]. The proton spin content has been chosen as $\left\langle S_{p}\right\rangle=0.441$ [41]. Exclusion limits from direct detection searches are presented : COUPP [36], KIMS 37], NAIAD [38], Picasso 39] and Xenon10 [40]. Exclusion limits obtained with neutrino telescopes (Super K 43]) are also displayed. The theoretical region, obtained within the framework of the constrained minimal supersymmetric model, is taken from 35]. Constraints from collider data and relic abundance $\Omega_{\chi} h^{2}$, as measured with WMAP 5year data [42], are accounted for. 


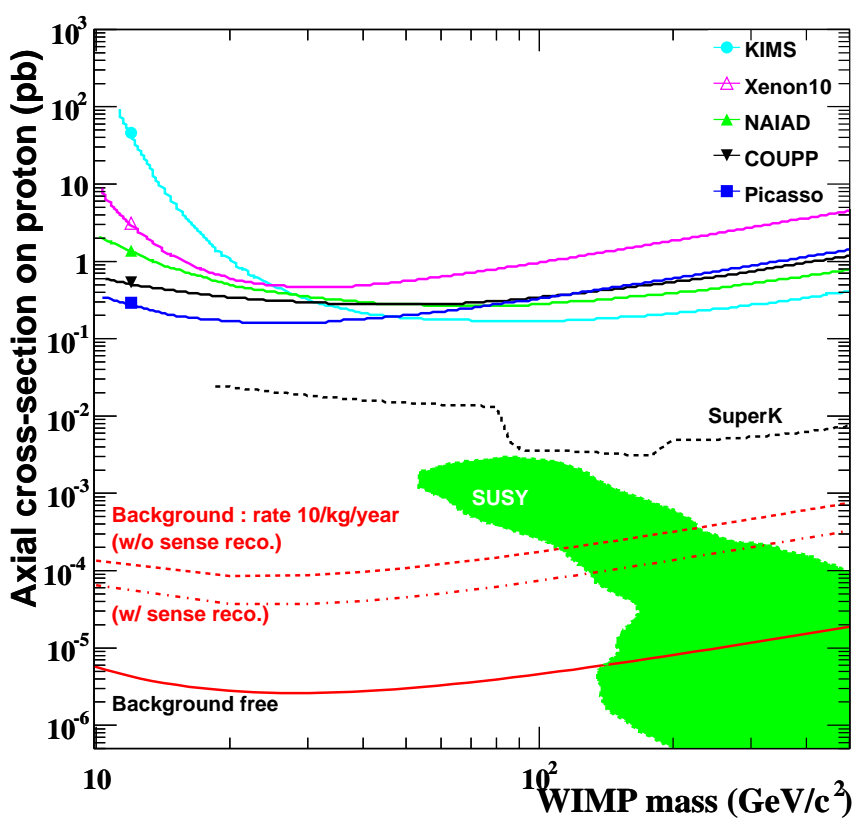

FIG. 11: Spin dependent cross section on proton (pb) as a function of the WIMP mass $\left(\mathrm{GeV} / \mathrm{c}^{2}\right)$. Results are presented in the case of pure-proton approximation [34]. The theoretical region, obtained within the framework of the constrained minimal supersymmetric model, is taken from 35]. Constraints from collider data and relic abundance are accounted for. Exclusion limits from direct and indirect Dark Matter searches are displayed (see text for details). The projected exclusion limit of a forthcoming directional detector (30 kg.year) is presented in three cases : background-free (solid line), with a background rate of 10 events $/ \mathrm{kg} /$ year with sense recognition (dot-dashed line) and without sense recognition (dashed line).

Several cases are considered on figure 11:

- the background-free measurement (dashed curve). It stands as the ultimate exclusion limit for a directional detector with such an exposure.

- with a background rate of 10 events/kg/year, which is large compared to current background measurements [3]. We consider the case of a directional detector with sense-recognition (dot-dashed line). The loss due to the presence of background is of the order of one order of magnitude, noticing that the use of the proposed Likelihood method allows the result to remain very satisfactory.

- with a background rate of 10 events $/ \mathrm{kg} /$ year but for a detector without sense-recognition (dashed line).
As stated above, as far as exclusion limits are concerned, the effect of not having sense-recognition is small.

It highlights the exclusion power of a rather light directional detector $(10 \mathrm{~kg})$, in the case that the discovery analysis [10] failed at recognizing the galactic origin of the signal, for instance if the axial nucleon-WIMP cross section is very low. Even with highly contaminated data or without sense recognition directional detection with a $10 \mathrm{~kg} \mathrm{CF}_{4}$ detector would allow to set very constraining exclusion limits, about 3 orders of magnitude better than the one imposed by existing direct detectors.

\section{CONCLUSION}

The conclusion of this study is threefold.

First, a new likelihood method to derive exclusion limits has been proposed for the directional search of galactic WIMPs. Only the angular part of the spectrum is taken into account (in a given energy range), arguing that the energy part of the WIMP spectrum is featureless and may even be mimic by the background one. On the same realistic set of directional data, the method has been shown to be more competitive than existing ones, or derived from existing ones.

Second, in the spirit of directional detector opimization, a study of the effect of detector configurations on the exclusion limits has been done. Comparing the results from the three methods shows that the proposed exclusion Likelihood method gives the best results in all cases. Moreover, the effect of not having sense recognition capability as well as having a low angular resolution has been shown to have little effect on exclusion limits. On the contrary, the effect of the energy threshold has been shown to largely influence the exclusion limits. This highlights the fact that the energy threshold is definitely the major experimental challenge for upcoming directional detectors, while no sense recognition and low angular resolution could be sufficient as far as exclusion limits are concerned, which might be the case for low exposure data. Note that this conclusion does not hold for a discovery strategy [10], e.g. if the unknown axial WIMP-nucleon cross section lies in the $10^{-3} \mathrm{pb}$ region. In this case, these detection issues remain of major interest, worth pursuing experimental efforts.

Third, the exclusion power of a light directional detector $(10 \mathrm{~kg})$ has been shown to be competitive with respect to the existing exclusion limits. Even with highly contaminated data or without sense recognition it allows to set exclusion limits about 3 orders of magnitude better than the one imposed by existing direct detectors. 
[3] E. Armengaud et al., Phys. Lett. B 687 (2010) 294-298

[4] D. N. Spergel, Phys. Rev. D 37 (1988) 1353.

[5] D. Santos et al., J. Phys. Conf. Ser. 65 (2007) 012012

[6] G. J. Alner et al., Nucl. Instr. Meth. A 555 (2005) 173.

[7] S. Ahlen et al., arXiv:1006.2928

[8] K. Miuchi et al., Phys. Lett. B 654 (2007) 58

[9] S. Ahlen et al., Int. J. Mod. Phys. A 25 (2010) 1

[10] J. Billard, F. Mayet, J. F. Macias-Perez and D. Santos, Phys. Lett. B 691 (2010) 156-162

[11] A. M. Green and B. Morgan, Phys. Rev. D 81 (2010) 061301

[12] S. Yellin, Phys. Rev. D 66 (2002) 032005

[13] S. Henderson, J. Monroe and P. Fisher, Phys. Rev. D 78 (2008) 015020

[14] S. Yellin, arXiv:0709.2701

[15] N. Bernal, A. Goudelis, Y. Mambrini, C. Munoz, JCAP 0901 (2009) 046

[16] A. M. Green, JCAP 0708 (2007) 022, JCAP 0807 (2008) 005

[17] G. Sciolla and C. J. Martoff, New J. Phys. 11 (2009) 105018

[18] A. M. Green and B. Morgan, Astropart. Phys. 27 (2007) 142 ,

[19] D. Dujmic et al., Nucl. Instrum. Meth. A 584 (2008) 327

[20] P. Majewski, D. Muna, D. P. Snowden-Ifft and N. J. C. Spooner, arXiv:0902.4430

[21] S. Burgos et al., arXiv:0809.1831

[22] B. Morgan, A. M. Green and N. J. C. Spooner, Phys. Rev. D 71 (2005) 103507,

[23] D. Santos et al., in preparation

[24] G. J. Feldman and R. D. Cousins, Phys. Rev. D 57 (1998) 3873

[25] G. Cowan, Statistical Data Analysis, Clarendon Press, Oxford, 1998.
[26] C. Grignon et al., arXiv:0909.0654

[27] C. J. Copi, L. M. Krauss, D. Simmons-Duffin and S. R. Stroiney, Phys. Rev. D 75 (2007) 023514

[28] A. Allaoua et al., Radiat. Meas. 44 (2009) 755

[29] A. M. Green, arXiv:1004.2383

[30] C. McCabe, arXiv:1005.0579

[31] C. Amsler et al. (Particle Data Group), Phys. Lett. B 667 (2008) 1

[32] P. Salucci, F. Nesti, G. Gentile and C. F. Martins, arXiv:1003.3101

[33] J. Billard, F. Mayet and D. Santos, in preparation

[34] D. R. Tovey et al., Phys. Lett. B 488 (2000) 17

[35] R. Trotta, F. Feroz, M. P. Hobson, L. Roszkowski and R. Ruiz de Austri, JHEP 0812 (2008) 024

[36] E. Behnke et al., Science 319 (2008) 933

[37] H. S. Lee et al., Phys. Rev. Lett. 99 (2007) 091301

[38] G. J. Alner et al., Phys. Lett. B 616 (2005) 17

[39] S. Archambault et al., Phys. Lett. B 682 (2009) 185

[40] J. Angle et al., Phys. Rev. Lett. 101 (2008) 091301

[41] A. F. Pacheco and D. Strottman, Phys. Rev. D 40 (1989) 2131-2133

[42] J. Dunkley et al., Astrophys. J. Suppl. 180 (2009) 306

[43] S. Desai et al., Phys. Rev. D 70 (2004) 083523

[44] J. D. Lewin \& P. F. Smith, Astropart. Physics 6 (1996) $87-112$

[45] Using the Born approximation, in the case of a spindependent interaction, the form factor is given by the Fourier transform of a thin shell [44] leading to: $F^{2}(5 \mathrm{keV})=0.99$ and $F^{2}(50 \mathrm{keV})=0.9$ in the case of a ${ }^{19} \mathrm{~F}$ target, justifying our approximation. Note that in the case of heavier targets, this approximation would be no longer valid. 